\title{
A SIMPLE PROOF OF CURVATURE ESTIMATE FOR CONVEX SOLUTION OF $k$-HESSIAN EQUATION
}

\author{
JIANCHUN CHU
}

\begin{abstract}
Guan-Ren-Wang 12 established the curvature estimate of convex hypersurface satisfying the Weingarten curvature equation $\sigma_{k}(\kappa(X))=$ $f(X, \nu(X))$. In this note, we give a simple proof of this result.
\end{abstract}

\section{INTRODUCTION}

Let $M \subset \mathbb{R}^{n+1}$ be a closed hypersurface. We consider the following curvature equation in a general form:

$$
\sigma_{k}(\kappa(X))=f(X, \nu(X)) \text { for } X \in M,
$$

where $\kappa(X)$ and $\nu(X)$ are principal curvatures and unit outer normal vector at $X \in M$, and $\sigma_{k}$ denotes the $k$-th elementary symmetric function

$$
\sigma_{k}(\kappa)=\sum_{i_{1}<i_{2}<\cdots<i_{k}} \kappa_{i_{1}} \kappa_{i_{2}} \cdots \kappa_{i_{k}}
$$

For $1 \leqslant k \leqslant n, \sigma_{k}(\kappa)$ are the Weingarten curvatures of $M$. In particular, $\sigma_{1}(\kappa), \sigma_{2}(\kappa)$ and $\sigma_{n}(\kappa)$ are the mean curvature, scalar curvature and Gauss curvature, respectively.

The curvature equation (1.1) plays a significant role in geometry. Many important geometric problem can be transformed into (1.1) with a special form of $f$, including the Minkowski problem $([15,16,17,6])$, the problem of prescribing general Weingarten curvature on outer normals by Alexandrov $([1,9])$, the problem of prescribing curvature measures in convex geometry $[2,16,11,10]$ ) and the prescribing curvature problem considered in $[3,22,5]$.

The curvature equation (1.1) has been studied extensively. When $k=1$, equation (1.1) is quasi-linear, so the curvature estimate follows from the classical theory of quasi-linear PDEs. When $k=n$, equation (1.1) is of Monge-Ampère type. The desired estimate was established by CaffarelliNirenberg-Spruck [4].

When $1<k<n$, if $f$ is independent of $\nu$, Caffarelli-Nirenberg-Spruck [5] established the curvature estimate; if $f$ depends only on $\nu$, the curvature estimate was proved by Guan-Guan [9]. In [13, 14, Ivochkina studied the Dirichlet problem of equation (1.1) on domains in $\mathbb{R}^{n}$ and obtained the curvature estimate under some additional assumptions on the dependence 
of $f$ on $\nu$. For the prescribing curvature measures problem, Guan-LinMa [11 and Guan-Li-Li [10] proved the curvature estimate for $f(X, \nu)=$ $\langle X, \nu\rangle \tilde{f}(X)$.

For general right-hand side $f(X, \nu)$, establishing the curvature estimate for equation (1.1) is very important and interesting in both geometry and PDEs. In [12, Guan-Ren-Wang solved the case $k=2$ (in [21, Spruck-Xiao gave a simplified proof). In [18, 19, Ren-Wang solved the cases $k=n-1$ and $k=n-2$. The other cases $2<k<n-2$ are still open. For general $k$, Guan-Ren-Wang [12] established the following curvature estimate for convex hypersurface:

Theorem 1.1. [Guan-Ren-Wang, [12, Theorem 1.1]] Let $M$ be a closed convex hypersurface satisfying curvature equation (1.1) for some positive function $f \in C^{2}(\Gamma)$, where $\Gamma$ is an open neighborhood of the unit normal bundle of $M$ in $\mathbb{R}^{n+1} \times \mathbb{S}^{n}$. There exists a constant $C$ depending only $n, k$, $\|M\|_{C^{1}}$, inf $f$ and $\|f\|_{C^{2}}$ such that

$$
\max _{X \in M, i=1,2, \cdots, n} \kappa_{i}(X) \leqslant C .
$$

In this note, we give a simple proof of Theorem 1.1. Compared to [12], we take a different approach. To establish the curvature estimate, the main difficulty is how to deal with the third order terms. We apply the maximum principle to a quantity involving the largest principal curvature $\kappa_{1}$, instead of the symmetric function of $\kappa$. This gives us more "good" third order terms, and so simplifies the argument.

\section{Preliminaries}

For any point $X_{0} \in M$, let $\left\{e_{i}\right\}_{i=1}^{n}$ be a local orthonormal frame near $X_{0}$ such that

$$
h_{i j}=\delta_{i j} \kappa_{i}, \quad \kappa_{1} \geqslant \kappa_{2} \geqslant \cdots \geqslant \kappa_{n} \text { at } X_{0} .
$$

We use the following notations:

$$
\sigma_{k}^{i j}=\frac{\partial \sigma_{k}}{\partial h_{i j}}, \quad \sigma_{k}^{i j, p q}=\frac{\partial^{2} \sigma_{k}}{\partial h_{i j} \partial h_{p q}} .
$$

Then at $X_{0}$, we have (see e.g. [8, 20])

$$
\sigma_{k}^{i j}=\sigma_{k-1}(\kappa \mid i) \delta_{i j}
$$

and

$$
\sigma_{k}^{i j, p q}= \begin{cases}\sigma_{k-2}(\kappa \mid i p) & \text { if } i=j, p=q, i \neq p \\ -\sigma_{k-2}(\kappa \mid i p) & \text { if } i=q, p=j, i \neq p \\ 0 & \text { otherwise, }\end{cases}
$$

where $\sigma_{s}\left(\kappa \mid i_{1} \cdots i_{r}\right)$ denotes $s$-th elementary symmetric function with $\kappa_{i_{1}}=$ $\kappa_{i_{2}}=\cdots=\kappa_{i_{r}}=0$. 
Here we list some well-known formulas:

Guass formula: $X_{i j}=-h_{i j} \nu$,

Weingarten equation : $\nu_{i}=h_{i j} e_{j}$,

Codazzi formula: $h_{i j p}=h_{i p j}$,

Guass equation : $R_{i j p q}=h_{i p} h_{j q}-h_{i q} h_{j p}$,

where $R_{i j p q}$ is the curvature tensor of $M$. We also have

$$
h_{p q i j}=h_{i j p q}+\left(h_{m q} h_{p j}-h_{m j} h_{p q}\right) h_{m i}+\left(h_{m q} h_{i j}-h_{m j} h_{i q}\right) h_{m p} .
$$

\section{Simple Proof of Theorem 1.1}

In this section, we give a simple proof of Theorem 1.1

Simple proof of Theorem 1.1. Since $M$ is convex, after shifting the origin of $\mathbb{R}^{n+1}$, we assume that $M$ is star-shaped with respect to the new origin. Thus the support function $u(X)=\langle X, \nu(X)\rangle$ is always positive. By assumptions, there exists a uniform constant $C>0$ such that

$$
\frac{1}{C} \leqslant u \leqslant C \text { for } X \in M \text {. }
$$

Let $\kappa_{1}$ be the largest principal curvature. Since $M$ is convex, to prove Theorem 1.1, it suffices to prove $\kappa_{1}$ is uniformly bounded from above. Without loss of generality, we assume that the set $\Omega=\left\{\kappa_{1}>0\right\}$ is not empty. On $\Omega$, we consider the following quantity

$$
Q=\log \kappa_{1}-A u,
$$

where $A>1$ is a constant to be determined later. Note that $Q$ is continuous on $\Omega$, and goes to $-\infty$ on $\partial \Omega$. Hence $Q$ achieves a maximum at a point $X_{0}$ with $\kappa_{1}\left(X_{0}\right)>0$. However, the function $Q$ may be not smooth at $X_{0}$ when the eigenspace of $\kappa_{1}$ has dimension strictly larger than 1, i.e., $\kappa_{1}=\kappa_{2}$ at $X_{0}$. To deal with this case, we apply the standard perturbation argument. Let $g$ be the first fundamental form of $M$ and $D$ be the corresponding Levi-Civita connection. We choose a local orthonormal frame $\left\{e_{i}\right\}_{i=1}^{n}$ near $X_{0}$ such that

$$
D_{e_{i}} e_{j}=0, \quad h_{i j}=\delta_{i j} \kappa_{i}, \quad \kappa_{1} \geqslant \kappa_{2} \geqslant \cdots \geqslant \kappa_{n} \text { at } X_{0} .
$$

We now apply a perturbation argument. Near $X_{0}$, we define a new tensor $B$ by

$$
B\left(V_{1}, V_{2}\right)=g\left(V_{1}, V_{2}\right)-g\left(V_{1}, e_{1}\right) g\left(V_{2}, e_{1}\right),
$$

for tangent vectors $V_{1}$ and $V_{2}$. Let $B_{i j}=B\left(e_{i}, e_{j}\right)$. It is clear that

$$
B_{i j}=\delta_{i j} B_{i i}, \quad B_{11}=0, \quad B_{i i}=1 \text { for } i>1 .
$$

We define the matrix by $\tilde{h}_{i j}=h_{i j}-B_{i j}$, and denote its eigenvalues by $\tilde{\kappa}_{1} \geqslant \tilde{\kappa}_{2} \geqslant \cdots \geqslant \tilde{\kappa}_{n}$. It then follows that $\kappa_{1} \geqslant \tilde{\kappa}_{1}$ near $X_{0}$ and

$$
\tilde{\kappa}_{i}=\left\{\begin{array}{ll}
\kappa_{1} & \text { if } i=1, \\
\kappa_{i}-1 & \text { if } i>1,
\end{array} \text { at } X_{0} .\right.
$$


Thus $\tilde{\kappa}_{1}>\tilde{\kappa}_{2}$ at $X_{0}$, which implies that $\tilde{\kappa}_{1}$ is smooth at $X_{0}$. We consider the perturbed quantity $\tilde{Q}$ defined by

$$
\tilde{Q}=\log \tilde{\kappa}_{1}-A u,
$$

which still achieves a local maximum at $X_{0}$. From now on, all the calculations will be carried out at $X_{0}$. For any $1 \leqslant i \leqslant n$, since $\tilde{\kappa}_{1}=\kappa_{1}$ at $X_{0}$, we have

$$
0=\tilde{Q}_{i}=\frac{\tilde{\kappa}_{1, i}}{\tilde{\kappa}_{1}}-A u_{i}=\frac{\tilde{\kappa}_{1, i}}{\kappa_{1}}-A u_{i}
$$

and

$$
0 \geqslant \sigma_{k}^{i i} \tilde{Q}_{i i}=\sigma_{k}^{i i}\left(\log \tilde{\kappa}_{1}\right)_{i i}-A \sigma_{k}^{i i} u_{i i} .
$$

In the following lemma, we estimate each term in (3.2) and obtain an inequality.

Lemma 3.1. At $X_{0}$, we have

$$
\begin{aligned}
0 \geqslant & 2 \sum_{p>1} \frac{\sigma_{k}^{11, p p} h_{11 p}^{2}}{\kappa_{1}}+2 \sum_{p>1} \frac{\sigma_{k}^{11} h_{11 p}^{2}}{\kappa_{1}\left(\kappa_{1}-\tilde{\kappa}_{p}\right)}-\frac{\sigma_{k}^{p p, q q} h_{p p 1} h_{q q 1}}{\kappa_{1}} \\
& +2 \sum_{p>1} \frac{\sigma_{k}^{p p} h_{p p 1}^{2}}{\kappa_{1}\left(\kappa_{1}-\tilde{\kappa}_{p}\right)}-\frac{\sigma_{k}^{p p} h_{11 p}^{2}}{\kappa_{1}^{2}}+\left(\frac{A}{C}-C\right) \sigma_{k}^{i i} h_{i i}^{2}-C A .
\end{aligned}
$$

Proof. First, let us recall the first and second derivatives of $\tilde{\kappa}_{1}$ at $X_{0}$ (see e.g. [20]):

$$
\begin{aligned}
\tilde{\kappa}_{1}^{p q} & :=\frac{\partial \tilde{\kappa}_{1}}{\partial \tilde{h}_{p q}}=\delta_{1 p} \delta_{1 q}, \\
\tilde{\kappa}_{1}^{p q, r s} & :=\frac{\partial^{2} \tilde{\kappa}_{1}}{\partial \tilde{h}_{p q} \partial \tilde{h}_{r s}}=\left(1-\delta_{1 p}\right) \frac{\delta_{1 q} \delta_{1 r} \delta_{p s}}{\tilde{\kappa}_{1}-\tilde{\kappa}_{p}}+\left(1-\delta_{1 r}\right) \frac{\delta_{1 s} \delta_{1 p} \delta_{q r}}{\tilde{\kappa}_{1}-\tilde{\kappa}_{r}} .
\end{aligned}
$$

We compute

$$
\begin{aligned}
\tilde{\kappa}_{1, i} & =\tilde{\kappa}_{1}^{p q} \tilde{h}_{p q i}=\tilde{h}_{11 i} \\
\tilde{\kappa}_{1, i i} & =\tilde{\kappa}_{1}^{p q} \tilde{h}_{p q i i}+\tilde{\kappa}_{1}^{p q, r s} \tilde{h}_{p q i} \tilde{h}_{r s i}=\tilde{h}_{11 i i}+2 \sum_{p>1} \frac{\tilde{h}_{1 p i}^{2}}{\kappa_{1}-\tilde{\kappa}_{p}},
\end{aligned}
$$

where we used $\tilde{\kappa}_{1}=\kappa_{1}$ at $X_{0}$. Using the definition of tensor $B$ and $\left(D_{e_{i}} e_{j}\right)\left(X_{0}\right)=0$, we see that

$$
B_{i j, p}=0, \quad B_{11, i i}=0 \text { at } X_{0} .
$$

Combining this with $\tilde{h}_{i j}=h_{i j}-B_{i j}$, we obtain

$$
\tilde{h}_{i j p}=h_{i j p}, \quad \tilde{h}_{11 i i}=h_{11 i i} \text { at } X_{0} .
$$

It then follows that

$$
\tilde{\kappa}_{1, i}=h_{11 i}, \quad \tilde{\kappa}_{1, i i}=h_{11 i i}+2 \sum_{p>1} \frac{h_{1 p i}^{2}}{\kappa_{1}-\tilde{\kappa}_{p}} .
$$


For the term $\sigma_{k}^{i i}\left(\log \tilde{\kappa}_{1}\right)_{i i}$ in $(\underline{3.2})$, using (3.3) and $\tilde{\kappa}_{1}=\kappa_{1}$ at $X_{0}$, we compute

$$
\begin{aligned}
\sigma_{k}^{i i}\left(\log \tilde{\kappa}_{1}\right)_{i i} & =\frac{\sigma_{k}^{i i} \tilde{\kappa}_{1, i i}}{\tilde{\kappa}_{1}}-\frac{\sigma_{k}^{i i} \tilde{\kappa}_{1, i}^{2}}{\tilde{\kappa}_{1}^{2}} \\
& =\frac{\sigma_{k}^{i i} h_{11 i i}}{\kappa_{1}}+2 \sum_{p>1} \frac{\sigma_{k}^{i i} h_{1 p i}^{2}}{\kappa_{1}\left(\kappa_{1}-\tilde{\kappa}_{p}\right)}-\frac{\sigma_{k}^{i i} h_{11 i}^{2}}{\kappa_{1}^{2}} .
\end{aligned}
$$

By (2.1), we have

$$
\begin{aligned}
\sigma_{k}^{i i} h_{11 i i} & =\sigma_{k}^{i i} h_{i i 11}+\sigma_{k}^{i i}\left(h_{i 1}^{2}-h_{i i} h_{11}\right) h_{i i}+\sigma_{k}^{i i}\left(h_{11} h_{i i}-h_{i 1}^{2}\right) h_{11} \\
& =\sigma_{k}^{i i} h_{i i 11}-\sigma_{k}^{i i} h_{i i}^{2} h_{11}+\sigma_{k}^{i i} h_{i i} h_{11}^{2} \\
& =\sigma_{k}^{i i} h_{i i 11}-\sigma_{k}^{i i} h_{i i}^{2} h_{11}+k f h_{11}^{2} .
\end{aligned}
$$

where we used

$$
\sum_{i} \sigma_{k}^{i i} h_{i i}=\sum_{i} \kappa_{i} \sigma_{k-1}(\kappa \mid i)=k \sigma_{k}(\kappa)=k f .
$$

On the other hand, differentiating (1.1) twice, we obtain

$$
\sigma_{k}^{i i} h_{i i 11} \geqslant-\sigma_{k}^{i j, p q} h_{i j 1} h_{p q 1}+\sum_{p} h_{p 11}\left(d_{\nu} f\right)\left(e_{p}\right)-C h_{11}^{2}-C .
$$

Combining this with (3.5),

$$
\sigma_{k}^{i i} h_{11 i i} \geqslant-\sigma_{k}^{i j, p q} h_{i j 1} h_{p q 1}+\sum_{p} h_{p 11}\left(d_{\nu} f\right)\left(e_{p}\right)-\sigma_{k}^{i i} h_{i i}^{2} h_{11}-C h_{11}^{2}-C .
$$

Substituting this into (3.4),

$$
\begin{aligned}
\sigma_{k}^{i i}\left(\log \tilde{\kappa}_{1}\right)_{i i} \geqslant & -\frac{\sigma_{k}^{i j, p q} h_{i j 1} h_{p q 1}}{\kappa_{1}}+2 \sum_{p>1} \frac{\sigma_{k}^{i i} h_{1 p i}^{2}}{\kappa_{1}\left(\kappa_{1}-\tilde{\kappa}_{p}\right)}-\frac{\sigma_{k}^{p p} h_{11 p}^{2}}{\kappa_{1}^{2}} \\
& +\frac{1}{\kappa_{1}} \sum_{p} h_{p 11}\left(d_{\nu} f\right)\left(e_{p}\right)-\sigma_{k}^{i i} h_{i i}^{2}-C h_{11}-C,
\end{aligned}
$$

assuming without loss of generality that $\kappa_{1} \geqslant 1$.

By Guass formula, Weingarten equation and Codazzi formula, we see that

$$
u_{i i}=\sum_{p} h_{i i p}\left\langle e_{p}, X\right\rangle-u h_{i i}^{2}+h_{i i}
$$

For the term $-A \sigma_{k}^{i i} u_{i i}$ in (3.2), we compute

$$
\begin{aligned}
-A \sigma_{k}^{i i} u_{i i} & =-A \sum_{p} \sigma_{k}^{i i} h_{i i p}\left\langle e_{p}, X\right\rangle+A u \sigma_{k}^{i i} h_{i i}^{2}-A \sigma_{k}^{i i} h_{i i} \\
& \geqslant-A \sum_{p} \sigma_{k}^{i i} h_{i i p}\left\langle e_{p}, X\right\rangle+\frac{A \sigma_{k}^{i i} h_{i i}^{2}}{C}-A k f,
\end{aligned}
$$


where we used $u \geqslant \frac{1}{C}$ and (3.6). Differentiating (1.1), we obtain

$$
\sigma_{k}^{i i} h_{i i p}=h_{p p}\left(d_{\nu} f\right)\left(e_{p}\right)+\left(d_{X} f\right)\left(e_{p}\right) .
$$

Substituting this into (3.8), we have

$$
-A \sigma_{k}^{i i} u_{i i} \geqslant-A \sum_{p} h_{p p}\left(d_{\nu} f\right)\left(e_{p}\right)\left\langle e_{p}, X\right\rangle+\frac{A \sigma_{k}^{i i} h_{i i}^{2}}{C}-C A .
$$

Combining (3.2), (3.7) and (3.9), we obtain

$$
\begin{aligned}
0 \geqslant & F^{i i} \tilde{Q}_{i i} \\
\geqslant & -\frac{\sigma_{k}^{i j, p q} h_{i j 1} h_{p q 1}}{\kappa_{1}}+2 \sum_{p>1} \frac{\sigma_{k}^{i i} h_{1 p i}^{2}}{\kappa_{1}\left(\kappa_{1}-\tilde{\kappa}_{p}\right)}-\frac{\sigma_{k}^{p p} h_{11 p}^{2}}{\kappa_{1}^{2}} \\
& +\frac{1}{\kappa_{1}} \sum_{p} h_{p 11}\left(d_{\nu} f\right)\left(e_{p}\right)-A \sum_{p} h_{p p}\left(d_{\nu} f\right)\left(e_{p}\right)\left\langle e_{p}, X\right\rangle \\
& +\left(\frac{A}{C}-1\right) \sigma_{k}^{i i} h_{i i}^{2}-C h_{11}-C A .
\end{aligned}
$$

Using (3.1), (3.3) and $u_{p}=h_{p p}\left\langle e_{p}, X\right\rangle$, for $1 \leqslant p \leqslant n$, we have

$$
\frac{h_{11 p}}{\kappa_{1}}-A h_{p p}\left\langle e_{p}, X\right\rangle=0 .
$$

Combining this with Codazzi formula, it is clear that

$$
\frac{1}{\kappa_{1}} \sum_{p} h_{p 11}\left(d_{\nu} f\right)\left(e_{p}\right)-A \sum_{p} h_{p p}\left(d_{\nu} f\right)\left(e_{p}\right)\left\langle e_{p}, X\right\rangle=0 .
$$

By [7, Lemma 3.1], we have

$$
\kappa_{1}=h_{11} \leqslant C \sigma_{k}^{11} h_{11}^{2} .
$$

Substituting (3.12) and (3.13) into (3.10),

$0 \geqslant-\frac{\sigma_{k}^{i j, p q} h_{i j 1} h_{p q 1}}{\kappa_{1}}+2 \sum_{p>1} \frac{\sigma_{k}^{i i} h_{1 p i}^{2}}{\kappa_{1}\left(\kappa_{1}-\tilde{\kappa}_{p}\right)}-\frac{\sigma_{k}^{p p} h_{11 p}^{2}}{\kappa_{1}^{2}}+\left(\frac{A}{C}-C\right) \sigma_{k}^{i i} h_{i i}^{2}-C A$.

Combining this with

$$
\begin{aligned}
& -\frac{\sigma_{k}^{i j, p q} h_{i j 1} h_{p q 1}}{\kappa_{1}}+2 \sum_{p>1} \frac{\sigma_{k}^{i i} h_{1 p i}^{2}}{\kappa_{1}\left(\kappa_{1}-\tilde{\kappa}_{p}\right)} \\
\geqslant & -\frac{\sigma_{k}^{p p, q q} h_{p p 1} h_{q q 1}}{\kappa_{1}}+2 \sum_{p>1} \frac{\sigma_{k}^{11, p p} h_{11 p}^{2}}{\kappa_{1}}+2 \sum_{p>1} \frac{\sigma_{k}^{11} h_{1 p 1}^{2}}{\kappa_{1}\left(\kappa_{1}-\tilde{\kappa}_{p}\right)}+2 \sum_{p>1} \frac{\sigma_{k}^{p p} h_{1 p p}^{2}}{\kappa_{1}\left(\kappa_{1}-\tilde{\kappa}_{p}\right)}
\end{aligned}
$$

and Codazzi formula, we obtain Lemma 3.1.

Lemma 3.2. At $X_{0}$, we have

$$
\sum_{p>1} \frac{\sigma_{k}^{p p} h_{11 p}^{2}}{\kappa_{1}^{2}} \leqslant 2 \sum_{p>1} \frac{\sigma_{k}^{11, p p} h_{11 p}^{2}}{\kappa_{1}}+2 \sum_{p>1} \frac{\sigma_{k}^{11} h_{11 p}^{2}}{\kappa_{1}\left(\kappa_{1}-\tilde{\kappa}_{p}\right)},
$$


assuming without loss of generality that $\kappa_{1} \geqslant 1$.

Proof. We define

$$
I=\left\{p \in\{2,3, \cdots, n\} \mid \kappa_{i}=\kappa_{1}\right\} .
$$

For $p \in I$, we have $\sigma_{k}^{p p}=\sigma_{k}^{11}$ and $\kappa_{1}-\tilde{\kappa}_{p}=1$. Thus,

$$
\sum_{p \in I} \frac{\sigma_{k}^{p p} h_{11 p}^{2}}{\kappa_{1}^{2}}=\frac{1}{\kappa_{1}} \sum_{p \in I} \frac{\sigma_{k}^{11} h_{11 p}^{2}}{\kappa_{1}} \leqslant \sum_{p \in I} \frac{\sigma_{k}^{11} h_{11 p}^{2}}{\kappa_{1}\left(\kappa_{1}-\tilde{\kappa}_{p}\right)} .
$$

For $p \notin I$, since $\tilde{\kappa}_{p}=\kappa_{p}-1$ and $\kappa_{p}>0$, then

$$
\kappa_{1}-\tilde{\kappa}_{p}=\kappa_{1}-\kappa_{p}+1 \leqslant \kappa_{1}+1 \leqslant 2 \kappa_{1}
$$

which implies

$$
\sum_{p \notin I} \frac{\sigma_{k}^{11} h_{11 p}^{2}}{\kappa_{1}^{2}} \leqslant 2 \sum_{p \notin I} \frac{\sigma_{k}^{11} h_{11 p}^{2}}{\kappa_{1}\left(\kappa_{1}-\tilde{\kappa}_{p}\right)}
$$

On the other hand, using $0<\kappa_{p}<\kappa_{1}$, we have

$$
\frac{\sigma_{k}^{p p}-\sigma_{k}^{11}}{\kappa_{1}^{2}} \leqslant \frac{\sigma_{k}^{p p}-\sigma_{k}^{11}}{\kappa_{1}\left(\kappa_{1}-\kappa_{p}\right)}=\frac{\sigma_{k}^{11, p p}}{\kappa_{1}} .
$$

It then follows that

$$
\sum_{p \notin I} \frac{\left(\sigma_{k}^{p p}-\sigma_{k}^{11}\right) h_{11 p}^{2}}{\kappa_{1}^{2}} \leqslant \sum_{p \notin I} \frac{\sigma_{k}^{11, p p} h_{11 p}^{2}}{\kappa_{1}} .
$$

Combining (3.15) and (3.16), we obtain

$$
\sum_{p \notin I} \frac{\sigma_{k}^{p p} h_{11 p}^{2}}{\kappa_{1}^{2}} \leqslant 2 \sum_{p \notin I} \frac{\sigma_{k}^{11} h_{11 p}^{2}}{\kappa_{1}\left(\kappa_{1}-\tilde{\kappa}_{p}\right)}+\sum_{p \notin I} \frac{\sigma_{k}^{11, p p} h_{11 p}^{2}}{\kappa_{1}} .
$$

Then Lemma 3.2 follows from (3.14) and (3.17).

The rest of the proof is very similar to [12, Theorem 1.1]. For the reader's convenience, we give all the details here.

Lemma 3.3. For $\varepsilon, \delta \in\left(0, \frac{1}{2}\right)$ and $1 \leqslant l \leqslant k-1$, there exists a uniform constant $\delta^{\prime}$ depending on $\varepsilon$ and $\delta$ such that if $\kappa_{l} \geqslant \delta \kappa_{1}$ and $\kappa_{l+1} \leqslant \delta^{\prime} \kappa_{1}$, then

$$
(1-2 \varepsilon) \frac{\sigma_{k}^{11} h_{111}^{2}}{\kappa_{1}^{2}} \leqslant-\frac{\sigma_{k}^{p p, q q} h_{p p 1} h_{q q 1}}{\kappa_{1}}+2 \sum_{p>1} \frac{\sigma_{k}^{p p} h_{p p 1}^{2}}{\kappa_{1}\left(\kappa_{1}-\tilde{\kappa}_{p}\right)}+C \kappa_{1},
$$

for some uniform constant $C$. 
Proof. Using [12, (2.4)] (see also [10, Lemma 3.2]), we have

$$
\begin{aligned}
& -\frac{\sigma_{k}^{p p, q q} h_{p p 1} h_{q q 1}}{\kappa_{1}}+\frac{\left(\sum_{p} \sigma_{k}^{p p} h_{p p 1}\right)^{2}}{\kappa_{1} \sigma_{k}} \\
\geqslant & \frac{\sigma_{k}}{\kappa_{1} \sigma_{l}^{2}}\left[\left(\sum_{p} \sigma_{l}^{p p} h_{p p 1}\right)^{2}-\sigma_{l} \sigma_{l}^{p p, q q} h_{p p 1} h_{q q 1}\right] \\
\geqslant & \frac{\sigma_{k}}{\kappa_{1} \sigma_{l}^{2}}\left[\sum_{p}\left(\sigma_{l}^{p p} h_{p p 1}\right)^{2}+\sum_{p \neq q}\left(\sigma_{l}^{p p} \sigma_{l}^{q q}-\sigma_{l} \sigma_{l}^{p p, q q}\right) h_{p p 1} h_{q q 1}\right] .
\end{aligned}
$$

Differentiating (1.1), we have

$$
\sum_{p} \sigma_{k}^{p p} h_{p p 1}=h_{11}\left(d_{\nu} f\right)\left(e_{1}\right)+\left(d_{X} f\right)\left(e_{1}\right)
$$

which implies

$$
\frac{\left(\sum_{p} \sigma_{k}^{p p} h_{p p 1}\right)^{2}}{\kappa_{1} \sigma_{k}}=\frac{\left(h_{11}\left(d_{\nu} f\right)\left(e_{1}\right)+\left(d_{X} f\right)\left(e_{1}\right)\right)^{2}}{\kappa_{1} f} \leqslant C \kappa_{1},
$$

assuming without loss of generality that $\kappa_{1} \geqslant 1$. Thus,

$$
\begin{aligned}
& -\frac{\sigma_{k}^{p p, q q} h_{p p 1} h_{q q 1}}{\kappa_{1}}+C \kappa_{1} \\
\geqslant & \frac{\sigma_{k}}{\kappa_{1} \sigma_{l}^{2}}\left[\sum_{p}\left(\sigma_{l}^{p p} h_{p p 1}\right)^{2}+\sum_{p \neq q}\left(\sigma_{l}^{p p} \sigma_{l}^{q q}-\sigma_{l} \sigma_{l}^{p p, q q}\right) h_{p p 1} h_{q q 1}\right] .
\end{aligned}
$$

We claim

$$
\begin{aligned}
& \sum_{p \neq q}\left(\sigma_{l}^{p p} \sigma_{l}^{q q}-\sigma_{l} \sigma_{l}^{p p, q q}\right) h_{p p 1} h_{q q 1} \\
\geqslant & -\varepsilon \sum_{p \leqslant l}\left(\sigma_{l}^{p p} h_{p p 1}\right)^{2}-\frac{C}{\varepsilon} \sum_{p>l}\left(\sigma_{l}^{p p} h_{p p 1}\right)^{2} .
\end{aligned}
$$

When $l=1$, we have $\sigma_{1}^{p p, q q}=0$. Then the claim (3.19) follows from the Cauchy-Schwarz inequality. When $l>1$, we split the left-handed side of 
(3.19) into three terms:

$$
\begin{aligned}
& \sum_{p \neq q}\left(\sigma_{l}^{p p} \sigma_{l}^{q q}-\sigma_{l} \sigma_{l}^{p p, q q}\right) h_{p p 1} h_{q q 1} \\
= & \sum_{p \neq q ; p, q \leqslant l}\left(\sigma_{l}^{p p} \sigma_{l}^{q q}-\sigma_{l} \sigma_{l}^{p p, q q}\right) h_{p p 1} h_{q q 1} \\
& +2 \sum_{p \leqslant l ; q>l}\left(\sigma_{l}^{p p} \sigma_{l}^{q q}-\sigma_{l} \sigma_{l}^{p p, q q}\right) h_{p p 1} h_{q q 1} \\
& +\sum_{p \neq q ; p, q>l}\left(\sigma_{l}^{p p} \sigma_{l}^{q q}-\sigma_{l} \sigma_{l}^{p p, q q}\right) h_{p p 1} h_{q q 1} \\
=: & T_{1}+T_{2}+T_{3} .
\end{aligned}
$$

By direct calculation and Newton's inequality, for $p \neq q$, we obtain (see [12, $(4.22)])$

$$
\sigma_{l}^{p p} \sigma_{l}^{q q}-\sigma_{l} \sigma_{l}^{p p, q q}=\sigma_{l-1}^{2}(\kappa \mid p q)-\sigma_{l}(\kappa \mid p q) \sigma_{l-2}(\kappa \mid p q) \geqslant 0 .
$$

Thus, for the term $T_{1}$ in (3.20), we have

$$
T_{1} \geqslant-\sum_{p \neq q ; p, q \leqslant l} \sigma_{l-1}^{2}(\kappa \mid p q)\left|h_{p p 1} h_{q q 1}\right| .
$$

For $p \neq q$ and $p, q \leqslant l$, since $\kappa_{p}, \kappa_{q} \geqslant \kappa_{l} \geqslant \delta \kappa_{1}, \kappa_{l+1} \leqslant \delta^{\prime} \kappa_{1}$ and $\kappa_{i}>0$ for all $i$, then

$$
\sigma_{l-1}(\kappa \mid p q) \leqslant \frac{C \kappa_{1} \cdots \kappa_{l+1}}{\kappa_{p} \kappa_{q}} \leqslant \frac{C \kappa_{l+1}}{\kappa_{q}} \cdot \frac{\kappa_{1} \cdots \kappa_{l}}{\kappa_{p}} \leqslant \frac{C \delta^{\prime} \sigma_{l}^{p p}}{\delta} .
$$

Similarly, we have

$$
\sigma_{l-1}(\kappa \mid p q) \leqslant \frac{C \delta^{\prime} \sigma_{l}^{q q}}{\delta}
$$

Choosing $\delta^{\prime}$ sufficiently small,

$$
\sigma_{l-1}^{2}(\kappa \mid p q) \leqslant\left(\frac{C \delta^{\prime}}{\delta}\right)^{2} \sigma_{l}^{p p} \sigma_{l}^{q q} \leqslant \frac{\varepsilon \sigma_{l}^{p p} \sigma_{l}^{q q}}{2}
$$

It then follows that

$$
T_{1} \geqslant-\frac{\varepsilon}{2} \sum_{p \neq q ; p, q \leqslant l}\left|\sigma_{l}^{p p} h_{p p 1}\right| \cdot\left|\sigma_{l}^{q q} h_{q q 1}\right| \geqslant-\frac{\varepsilon}{2} \sum_{p \leqslant l}\left(\sigma_{l}^{p p} h_{p p 1}\right)^{2} .
$$

For the terms $T_{2}$ and $T_{3}$ in (3.20), using (3.21) and the Cauchy-Schwarz inequality,

$$
\begin{aligned}
T_{2}+T_{3} & \geqslant-2 \sum_{p \leqslant l ; q>l} \sigma_{l}^{p p} \sigma_{l}^{q q}\left|h_{p p 1} h_{q q 1}\right|-\sum_{p \neq q ; p, q>l} \sigma_{l}^{p p} \sigma_{l}^{q q}\left|h_{p p 1} h_{q q 1}\right| \\
& \geqslant-\frac{\varepsilon}{2} \sum_{p \leqslant l}\left(\sigma_{l}^{p p} h_{p p 1}\right)^{2}-\frac{C}{\varepsilon} \sum_{p>l}\left(\sigma_{l}^{p p} h_{p p 1}\right)^{2} .
\end{aligned}
$$

Substituting (3.22) and (3.23) into (3.20), we obtain the claim (3.19). 
Combining (3.18) and (3.19),

$$
\begin{aligned}
& (1-\varepsilon) \frac{\sigma_{k}\left(\sigma_{l}^{11}\right)^{2} h_{111}^{2}}{\kappa_{1} \sigma_{l}^{2}} \leqslant(1-\varepsilon) \frac{\sigma_{k}}{\kappa_{1} \sigma_{l}^{2}} \sum_{p \leqslant l}\left(\sigma_{l}^{p p} h_{p p 1}\right)^{2} \\
\leqslant & -\frac{\sigma_{k}^{p p, q q} h_{p p 1} h_{q q 1}}{\kappa_{1}}+\frac{C \sigma_{k}}{\varepsilon \kappa_{1} \sigma_{l}^{2}} \sum_{p>l}\left(\sigma_{l}^{p p} h_{p p 1}\right)^{2}+C \kappa_{1} .
\end{aligned}
$$

Since $\kappa_{i}>0$ for all $i$ and $\kappa_{l+1} \leqslant \delta^{\prime} \kappa_{1}$, we have

$$
\frac{\sigma_{k}}{\kappa_{1} \sigma_{k}^{11}}=\frac{\kappa_{1} \sigma_{k}^{11}+\sigma_{k}(\kappa \mid 1)}{\kappa_{1} \sigma_{k}^{11}} \geqslant 1
$$

and

$$
\frac{\kappa_{1} \sigma_{l}^{11}}{\sigma_{l}}=1-\frac{\sigma_{l}(\kappa \mid 1)}{\sigma_{l}} \geqslant 1-\frac{C \kappa_{2} \cdots \kappa_{l+1}}{\kappa_{1} \cdots \kappa_{l}}=1-\frac{C \kappa_{l+1}}{\kappa_{1}} \geqslant 1-C \delta^{\prime} .
$$

Thus, at the expense of decreasing $\delta^{\prime}$, we obtain

$$
\begin{aligned}
& (1-\varepsilon) \frac{\sigma_{k}\left(\sigma_{l}^{11}\right)^{2} h_{111}^{2}}{\kappa_{1} \sigma_{l}^{2}}=(1-\varepsilon) \frac{\sigma_{k}^{11}}{\kappa_{1}^{2}} \cdot \frac{\sigma_{k}}{\kappa_{1} \sigma_{k}^{11}} \cdot\left(\frac{\kappa_{1} \sigma_{l}^{11}}{\sigma_{l}}\right)^{2} h_{111}^{2} \\
\geqslant & (1-\varepsilon)\left(1-C \delta^{\prime}\right)^{2} \frac{\sigma_{k}^{11} h_{111}^{2}}{\kappa_{1}^{2}} \geqslant(1-2 \varepsilon) \frac{\sigma_{k}^{11} h_{111}^{2}}{\kappa_{1}^{2}} .
\end{aligned}
$$

On the other hand, using $\kappa_{l} \geqslant \delta \kappa_{1}$ and $\kappa_{i}>0$ for all $i$, for $p>l$, we have

$$
\frac{\sigma_{l}^{p p}}{\sigma_{l}} \leqslant \frac{C \kappa_{1} \cdots \kappa_{l-1}}{\kappa_{1} \cdots \kappa_{l}} \leqslant \frac{C}{\kappa_{l}} \leqslant \frac{C}{\delta \kappa_{1}} .
$$

This implies

$$
\frac{C \sigma_{k}}{\varepsilon \kappa_{1} \sigma_{l}^{2}} \sum_{p>l}\left(\sigma_{l}^{p p} h_{p p 1}\right)^{2}=\frac{C}{\varepsilon} \sum_{p>l}\left(\frac{\sigma_{l}^{p p}}{\sigma_{l}}\right)^{2} \cdot \frac{\sigma_{k} h_{p p 1}^{2}}{\kappa_{1}} \leqslant \frac{C}{\varepsilon \delta^{2}} \sum_{p>l} \frac{\sigma_{k} h_{p p 1}^{2}}{\kappa_{1}^{3}} .
$$

Since $\kappa_{l+1} \leqslant \delta^{\prime} \kappa_{1}$ and $\kappa_{i}>0$ for all $i$, then for $l<p \leqslant k$,

$$
\frac{\sigma_{k}}{\kappa_{1}} \leqslant \frac{\delta^{\prime} \sigma_{k}}{\kappa_{p}} \leqslant \frac{C \delta^{\prime} \kappa_{1} \cdots \kappa_{k}}{\kappa_{p}} \leqslant C \delta^{\prime} \sigma_{k}^{p p} .
$$

For $p>k$,

$$
\frac{\sigma_{k}}{\kappa_{1}} \leqslant \frac{\delta^{\prime} \sigma_{k}}{\kappa_{k}} \leqslant C \kappa_{1} \cdots \kappa_{k-1} \leqslant C \delta^{\prime} \sigma_{k}^{p p} .
$$

So $\frac{\sigma_{k}}{\kappa_{1}} \leqslant C \delta^{\prime} \sigma_{k}^{p p}$ for $p>l$. It then follows that

$$
\frac{C}{\varepsilon \delta^{2}} \sum_{p>l} \frac{\sigma_{k} h_{p p 1}^{2}}{\kappa_{1}^{3}} \leqslant \frac{C \delta^{\prime}}{\varepsilon \delta^{2}} \sum_{p>l} \frac{\sigma_{k}^{p p} h_{p p 1}^{2}}{\kappa_{1}^{2}} .
$$

Combining (3.26) and (3.27), and using $\kappa_{1}-\tilde{\kappa}_{i} \leqslant \kappa_{1}+1$ for $i>1$, at the expense of decreasing $\delta^{\prime}$, we see that

$$
\frac{C \sigma_{k}}{\varepsilon \kappa_{1} \sigma_{l}^{2}} \sum_{p>l}\left(\sigma_{l}^{p p} h_{p p 1}\right)^{2} \leqslant \sum_{p>l} \frac{\sigma_{k}^{p p} h_{p p 1}^{2}}{\kappa_{1}^{2}} \leqslant 2 \sum_{p>1} \frac{\sigma_{k}^{p p} h_{p p 1}^{2}}{\kappa_{1}\left(\kappa_{1}-\tilde{\kappa}_{p}\right)},
$$


assuming without loss of generality that $\kappa_{1} \geqslant 1$. Substituting (3.25) and (3.28) into (3.24), we obtain

$$
(1-2 \varepsilon) \frac{\sigma_{k}^{11} h_{111}^{2}}{\kappa_{1}^{2}} \leqslant-\frac{\sigma_{k}^{p p, q q} h_{p p 1} h_{q q 1}}{\kappa_{1}}+2 \sum_{p>1} \frac{\sigma_{k}^{p p} h_{p p 1}^{2}}{\kappa_{1}\left(\kappa_{1}-\tilde{\kappa}_{p}\right)}+C \kappa_{1},
$$

as required.

Lemma 3.4. For $\delta \in\left(0, \frac{1}{2}\right)$ and $1 \leqslant l \leqslant k-1$, there exists a uniform constant $\delta^{\prime}$ and $C$ depending on $\delta$ such that if $\kappa_{l} \geqslant \delta \kappa_{1}$ and $\kappa_{l+1} \leqslant \delta^{\prime} \kappa_{1}$, then $\kappa_{1} \leqslant C$.

Proof. Combining Lemma 3.1, 3.2 and 3.3, we obtain

$$
0 \geqslant-2 \varepsilon \frac{\sigma_{k}^{11} h_{111}^{2}}{\kappa_{1}^{2}}+\left(\frac{A}{C}-C\right) \sigma_{k}^{i i} h_{i i}^{2}-C \kappa_{1}-C A .
$$

Using (3.11), we have

$$
-2 \varepsilon \frac{\sigma_{k}^{11} h_{111}^{2}}{\kappa_{1}^{2}}=-2 \varepsilon A^{2} \sigma_{k}^{11} h_{11}^{2}\left\langle e_{1}, X\right\rangle^{2} \geqslant-C \varepsilon A^{2} \sigma_{k}^{11} h_{11}^{2} .
$$

It then follows that

$$
0 \geqslant\left(\frac{A}{C}-C-C \varepsilon A^{2}\right) \sigma_{k}^{i i} h_{i i}^{2}-C \kappa_{1}-C A .
$$

Using (3.13), we have

$$
0 \geqslant\left(\frac{A}{C_{0}}-C_{0}-C_{0} \varepsilon A^{2}\right) \kappa_{1}-C_{0} A
$$

for some uniform constant $C_{0}$. Choosing $A=2 C_{0}^{2}+C_{0}$ and $\varepsilon=\frac{1}{A^{2}}$, we obtain $\kappa_{1} \leqslant C$, as required.

We now complete the proof of Theorem 1.1. Set $\delta_{1}=\frac{1}{3}$, By Lemma 3.4, there exists $\delta_{2}$ such that if $\kappa_{2} \leqslant \delta_{2} \kappa_{1}$, then $\kappa_{1} \leqslant C$. If $\kappa_{2}>\delta_{2} \kappa_{1}$, using Lemma 3.4 again, there exists $\delta_{3}$ such that if $\kappa_{3} \leqslant \delta_{3} \kappa_{1}$, then $\kappa_{1} \leqslant C$. Repeating the above argument, we obtain $\kappa_{1} \leqslant C$ or $\kappa_{k}>\delta_{k} \kappa_{1}$. In the latter case, since $\kappa_{1} \geqslant \kappa_{2} \geqslant \cdots \geqslant \kappa_{k}>\delta_{k} \kappa_{1}$ and $\kappa_{i}>0$ for all $i$, then

$$
\delta_{k}^{k} \kappa_{1}^{k}<\kappa_{1} \cdots \kappa_{k} \leqslant \sigma_{k}=f \leqslant C,
$$

which implies $\kappa_{1} \leqslant C$, as required.

\section{REFERENCES}

[1] Aleksandrov, A. D. Uniqueness theorems for surfaces in the large. I, (Russian) Vestnik Leningrad. Univ. 11 (1956), no. 19, 5-17.

[2] Alexandroff, A. Existence and uniqueness of a convex surface with a given integral curvature, C. R. (Doklady) Acad. Sci. URSS (N.S.) 35 (1942). 131-134.

[3] Bakelman, I. Ja., Kantor, B. E. Existence of a hypersurface homeomorphic to the sphere in Euclidean space with a given mean curvature, (Russian) Geometry and topology, No. 1 (Russian), pp. 310. Leningrad. Gos. Ped. Inst. im. Gercena, Leningrad, 1974. 
[4] Caffarelli, L., Nirenberg, L., Spruck, J. The Dirichlet problem for nonlinear secondorder elliptic equations. I. Monge-Ampère equation, Comm. Pure Appl. Math. 37 (1984), no. 3, 369-402.

[5] Caffarelli, L., Nirenberg, L., Spruck, J. Nonlinear second order elliptic equations. IV. Starshaped compact Weingarten hypersurfaces, Current topics in partial differential equations, 126, Kinokuniya, Tokyo, 1986.

[6] Cheng, S. Y., Yau, S.-T. On the regularity of the solution of the $n$-dimensional Minkowski problem, Comm. Pure Appl. Math. 29 (1976), no. 5, 495-516.

[7] Chou, K.-S., Wang, X.-J. A variational theory of the Hessian equation, Comm. Pure Appl. Math. 54 (2001), no. 9, 1029-1064.

[8] Gerhardt, C. Closed Weingarten hypersurfaces in Riemannian manifolds, J. Differential Geom. 43 (1996), no. 3, 612-641.

[9] Guan, B., Guan, P. Convex hypersurfaces of prescribed curvatures, Ann. of Math. (2) 156 (2002), no. 2, 655-673.

[10] Guan, P., Li, J., Li, Y. Y. Hypersurfaces of prescribed curvature measure, Duke Math. J. 161 (2012), no. 10, 1927-1942.

[11] Guan, P., Lin, C., Ma, X.-N. The existence of convex body with prescribed curvature measures, Int. Math. Res. Not. IMRN 2009, no. 11, 1947-1975.

[12] Guan, P., Ren, C., Wang, Z. Global $C^{2}$-estimates for convex solutions of curvature equations, Comm. Pure Appl. Math. 68 (2015), no. 8, 1287-1325.

[13] Ivochkina, N. M. Solution of the Dirichlet problem for equations of mth order curvature, Mat. Sb. 180 (1989), no. 7, 867-887, 991; translation in Math. USSR-Sb. 67 (1990), no. 2, 317-339.

[14] Ivochkina, N. M. The Dirichlet problem for the curvature equation of order $m$, Algebra i Analiz 2 (1990), no. 3, 192-217; translation in Leningrad Math. J. 2 (1991), no. 3, 631-654.

[15] Nirenberg, L. The Weyl and Minkowski problems in differential geometry in the large, Comm. Pure Appl. Math. 6 (1953), 337-394.

[16] Pogorelov, A. V. On existence of a convex surface with a given sum of the principal radii of curvature, (Russian) Uspekhi Mat. Nauk. 8 (1953), 127-130.

[17] Pogorelov, A. V. The Minkowski multidimensional problem, Scripta Series in Mathematics. V. H. Winston \& Sons, Washington, D. C.; Halsted Press [John Wiley \& Sons], New York-Toronto-London, 1978.

[18] Ren, C., Wang, Z. On the curvature estimates for Hessian equations, Amer. J. Math. 141 (2019), no. 5, 1281-1315.

[19] Ren, C., Wang, Z. The global curvature estimate for the $n-2$ Hessian equation, preprint, arXiv: 2002.08702.

[20] Spruck, J. Geometric aspects of the theory of fully nonlinear elliptic equations, Global theory of minimal surfaces, 283-309, Clay Math. Proc., 2, Amer. Math. Soc., Providence, RI, 2005.

[21] Spruck, J., Xiao, L. A note on star-shaped compact hypersurfaces with prescribed scalar curvature in space forms, Rev. Mat. Iberoam. 33 (2017), no. 2, 547-554.

[22] Treibergs, A. E., Wei, S. W. Embedded hyperspheres with prescribed mean curvature, J. Differential Geom. 18 (1983), no. 3, 513-521.

Department of Mathematics, Northwestern University, 2033 Sheridan Road, EVANSTON, IL 60208

E-mail address: jianchun@math.northwestern.edu 